\title{
Ethnobotanical Study and Plant Diversity in the Forest of Kedarnath Valley, Garhwal Himalaya, India
}

\author{
Chandi Prasad $\dagger$, Ramesh C. Sharma and Rahul Kumar \\ Department of Environmental Sciences, H.N.B. Garhwal University (Central University), Srinagar, Garhwal-246174, \\ Uttarakhand, India \\ $\dagger$ Corresponding author: Chandi Prasad; cpsemwal2@gmail.com
}

Nat. Env. \& Poll. Tech.

Website: www.neptjournal.com

Received: 28-07-2020

Revised: 14-09-2021

Accepted: 15-10-2020

Key Words:

Forest resources

Kedarnath valley

Dominant species

Total basal area

\begin{abstract}
The present study was carried out in the forest of Kedarnath valley in Garhwal Himalaya. The aim of the study was to access the diversity status and ecological status. The study was conducted following the stratified sampling techniques by placing quadrates $(1 \mathrm{~m} \times 1 \mathrm{~m})$ for herbs, $(5 \mathrm{~m} \times 5 \mathrm{~m})$ for shrubs, and $(10 \mathrm{~m} \times 10 \mathrm{~m})$ size for trees in the forest area. A total number of 221 plant species were recorded during the floristic survey in the project area. Plant diversity of the project area encompasses 49 species of trees, 28 species of shrubs, and 144 species of herbs. Important value index, the Shannon diversity index, and total basal area species were recorded. The tree density in the present study was highest in the Kedarnath valley which ranged from 0.3 to 8.5 no./ha. Shrub density in the present study varied from 0.4 to 13.5 no./ha, whereas herb density ranged between 0.2 to 22.4 no.ha $^{-1}$. Total basal cover (TBC) for trees showed a range of 9.542 to $0.075 \mathrm{~m}^{2}$. ha ${ }^{-1}$, and the Shannon diversity index $(\bar{H})$ for tree species was recorded from a minimum of 0.976 to a maximum of 3.048. The horrific disaster in the Kedarnath valley in 2013 caused a lot of damage to the bugyals (High altitude grass) and forests of the valley. About 500 species of vesicular medicinal plants, fodder plants, and other important plant species were washed away (Botanical Survey of India 2015). The current study is a pioneer in the aspect and can be helpful in making district forest plans, protocols, and implementation of forest policy to protect the forest by local people.
\end{abstract}

\section{INTRODUCTION}

Forest plays a vital role in the sustenance of the Himalayan ecosystem. The mountain people are directly dependent on forest resources for food, firewood, fodder, and timber. Mountain forests are rich in biodiversity and are distributed according to different elevations and slopes. Forest also plays an important role in providing habitat for wildlife. The Kedarnath valley is an important upper stretch of the Ganga River system in the Uttarakhand Himalaya. Characterized by rugged, rough, and precipitous slopes, the entire valley is very prone to landslides, mass wasting, landslips, and slope failures.

The climate and vegetation of Uttarakhand vary greatly with different altitudes, from a glacier at a high altitude of $7,817 \mathrm{~m}$ asl. to a subtropical forest at lower altitudes. The high altitude region is covered by ice and bare rock. The annual rainfall is $1,550 \mathrm{~mm}$ and the average annual temperature ranges between $-8^{\circ} \mathrm{C}$ to $25^{\circ} \mathrm{C}$. The human population density of the state is 189 persons per $\mathrm{km}^{2}$, which is lower than the national average of 382 persons per $\mathrm{km}^{2}$ (Census 2011). According to the $19^{\text {th }}$ Livestock Census (2012), 4.79 million livestock population has been reported in Uttarakhand. The climate is subtropical in the south and temperate in the north. The climate remains cool in the middle zone of the state (Srivastava \& Singh 2005). The state represents one of the four high diversity states of the Indian Himalayan region with about 4,248 species of Angiosperms and 18 species in Gymnosperms (Srivastava \& Singh 2005).

The Uttarakhand area has been a major site plant exploration since 1796 when Thomas Hardwicke collected plants from the Alaknanda Valley of Garhwal Himalaya. By the beginning of the $21^{\text {st }}$ century, a large number of plant collectors have explored the area and a great deal of information was available about the flowering plants of this area. Based on these collections, floristic reports, and their own collections, Uniyal et al. (2007) compiled a checklist of flowering plants of Uttarakhand as baseline data for writing the flora of Uttarakhand. This valuable document suggests the presence of nearly 4,700 species of flowering plants, including 32 species of Gymnosperms and a few cultivated species. Kimothi et al. (1989) studied some medicinal plants of the Gopeshwar-Tungnath region of Uttar Pradesh. Negi et al. (2008) worked on the inventory of species richness of Panchayat forests and adjoining reserve forests in three dis- 
tricts of Garhwal Himalaya, India. Kumar (2009) identified major religious plants of Rudraprayag district (Garhwal), Uttarakhand (India). Semwal et al. (2010) studied medicinal plants used by local Vaidyas in Ukhimath block, Uttarakhand, India. Ballabha et al. (2013) studied community structure and plant diversity of community-based religious conserved forest of Garhwal Himalaya, India. Pala et al. (2016) worked on community structure and plant diversity of community-based religious conserved forest of Garhwal Himalaya, India. Nautiyal et al. (2017) studied the exploration of some important fodder plants of the Joshimath area of the Chamoli district of Garhwal, Uttarakhand. Singh et al. (2017) studied ethnomedicinal plants used by local inhabitants of Jakholi block, Rudraprayag district, western Himalaya, India. Prasad and Sharma (2018) studied wild edible plant resources of Kedarnath valley, Garhwal Himalaya, Uttarakhand.

The state of Uttarakhand is an important part of the Himalayas. Uttarakhand covers an area of $1.63 \%$ of the geographical area of India. The forest cover of Uttarakhand is $24,295 \mathrm{~km}^{2}$ which is $45.43 \%$ of the state's geographical area. In the term of forest canopy density classes, the state has $4,969 \mathrm{~km}^{2}$ under very dense forest, $12,884 \mathrm{~km}^{2}$ under moderately dense forest; and $6,442 \mathrm{~km}^{2}$ under open forest (FSI 2017). The forest in Uttarakhand is divided into sixteen types (FSI 2017), which are characterized by Northern Tropical Dry Deciduous Forests (Dry Sal-bearing Forest and Dry Plain Forest), Himalayan Sub-tropical Pine Forests (Himalayan Chir-pine and Sub-tropical Scrubs and Euphorbia Scrub), Himalayan Moist Temperate Forests (Lower Western Himalayan Temperate and Upper West Himalayan Temperate Forests), Himalayan Dry Temperate Forest (Dry Temperate Coniferous and West Himalayan Dry Juniper Forest), Sub-alpine Forests (West Himalayan Birch/Fir Forest and Pastures) and Moist and Dry Alpine Scrub Forests.

\section{MATERIALS AND METHODS}

\section{Study Area}

The Kedarnath valley is located between the coordinates of latitude $300^{\prime} 25^{\circ}$ " to $300^{\circ} 45^{\circ}$ " $\mathrm{N}$ and longitude $780^{\prime} 55^{\circ}$ " to 790 ' $20^{\circ}$ " E of Ukhimath tehsil in the Rudraprayag district of Garhwal Himalaya, Uttarakhand. The survey was done from a lower altitude of $864 \mathrm{~m}$ above m.s.l to the alpine meadow of Kedarnath-Tunganath (3,680-4,000 m above m.s.l). This study was carried out in nine study sites of Kedarnath valley of Ukhimath tehsil (Fig. 1), their locations, geographical coordinates, and elevations have been presented in Table 1 . The Kedarnath valley is in the district of Rudraprayag with an area of $1,248 \mathrm{~km}^{2}$ including 248 villages with a total population of 87,024 including 42,614 males and 44,410 females (Census of India 2011).

\section{Data Collection}

Information regarding the plant biodiversity, economically important plants, fruits and fodder plants and medicinal plants were collected. Field visits were made for the collection of plants and also to collect information on the biodiversity of the area. Plants were identified by the villagers, and scientific validation of these plants was made by the Himalayan Herbarium, Department of Botany and High Altitude Plant Physiology Research Center (HAPPRC), H.N.B. Garhwal University (A Central University), Srinagar-Garhwal. Relevant uses of these plants were also collected from different literature.

Plant biodiversity analysis was carried out during the study period when the majority of the plants were at the peak of their growth. In every study site, 10 transects of 10 $\mathrm{m} \times 10 \mathrm{~m}(100 \mathrm{sq} \mathrm{m})$ size was randomly laid to study tree species and 10 quadrates of $5 \mathrm{~m} \times 5 \mathrm{~m}(25 \mathrm{sq} \mathrm{m})$ size were randomly laid to study shrub species. The herbaceous species was studied by laying 10 quadrats of $1 \mathrm{~m} \times 1 \mathrm{~m}(1 \mathrm{sq} \mathrm{m})$ size randomly in each study site.

\section{Quantitative Analysis}

The important quantitative analysis such as density, frequency, and abundance of tree species, shrubs and herbs species were determined as per Gates (1949), Curtis and Mc-Intosh (1950), Misra and Puri (1954), Curtis (1951), Phillips (1959), Misra (1969), Mullar-Dombois and Ellenberg (1974).

Density:

$$
\text { Density }=\frac{\begin{array}{c}
\text { Total number of individuals of a } \\
\text { species in all quadrats }
\end{array}}{\text { Total number of quadrats studied }}
$$

(b) Frequency (\%):

Number of quadrats in which the

Frequency $(\%)=\frac{\text { species occurred } \times 100}{\text { Total number of quadrats studied }}$

(c) Abundance:

Total number of individuals of a

Abundance $=\frac{\text { species in all quadrats }}{\begin{array}{l}\text { Total number of quadrats in } \\ \text { which the species occurred }\end{array}}$

\section{Basal Area}

The basal area is the area of a given section of land that is occupied by the cross-section of tree trunks and stems at the base. The basal area per tree is the cross-sectional area of a tree at breast height. The term is used in forest manage- 

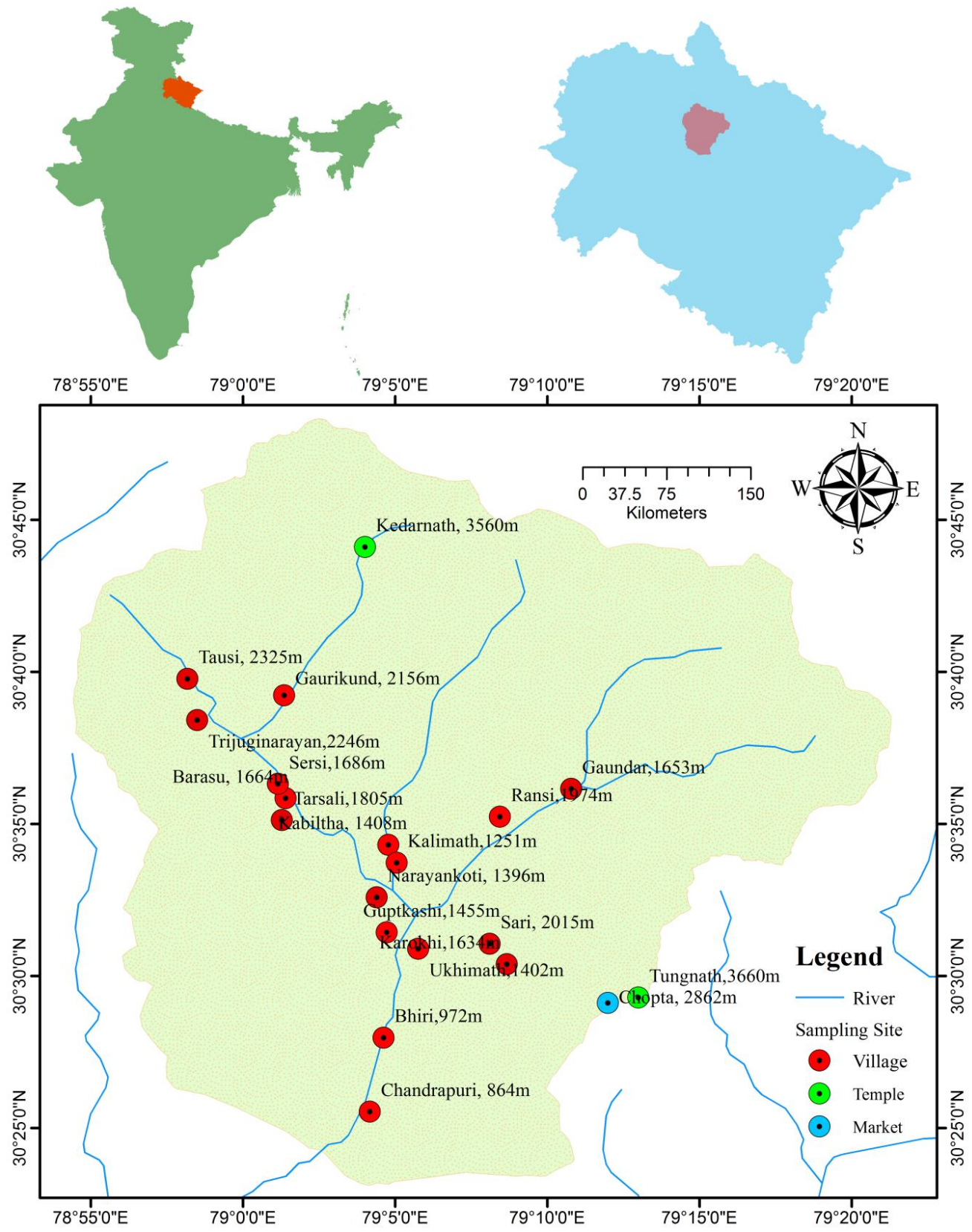

Fig. 1: Location map of the study area: The Kedarnath valley.

ment and forest ecology (Phillips 1959) as:

Sum of all

Mean of the circumference $(c)=\frac{\text { cbh }(\text { circumference })}{\text { Total number of species }}$

Mean Basal area $=\frac{C^{2}}{4 \pi}$
Total Basal area $=$ Mean Basal area $\times$ Density

Where, $\mathrm{cbh}=$ Circumference at breast height, $\mathrm{C}=$ sum of cbh value of all individuals of a tree species within each plot and $\pi=3.14$.

\section{Importance Value Index}

This index is used to determine the overall importance of 
each species in the community structure. In calculating this index, the percentage values of the relative frequency, relative density, and relative dominance are summed up together and this value is designated as the Importance Value Index or IVI of the species (Curtis 1959).

\section{(a) Relative density:}

Number of individual of the

$$
\text { Relative Density }=\frac{\text { species } \times 100}{\begin{array}{c}
\text { Number of individual of all the } \\
\text { species }
\end{array}}
$$

\section{(b) Relative frequency:}

Number of occurrence of the

$$
\text { Relative Frequency }=\frac{\text { species } \times 100}{\begin{array}{c}
\text { Number of occurrence of all the } \\
\text { species }
\end{array}}
$$

\section{(c) Relative dominance:}

Total basal area of the species Relative Dominance $=\frac{\times 100}{\text { Total basal area of all the species }}$

The total basal area was calculated from the sum of the total diameter of immerging stems. In trees, poles, and saplings, the basal area was measured at breast height $(1.5 \mathrm{~m})$ and by using the formula $\pi r^{2}$; but in the case of herbaceous vegetation it was measured on the ground level by using calipers.

Species diversity indices (Shannon Wiener Index) of general diversity $(\bar{H})$ was computed using the following formula:

Shannon Wiener Diversity Index $(\bar{H})=-\sum_{l-1}^{s}\left(\frac{n_{i}}{N}\right) \log _{2}\left(\frac{n_{i}}{N}\right)$
Where, $\bar{H}=$ Shannon Wiener index of diversity; $n_{i}=$ total no. of individuals of a species; and $\mathrm{N}=$ total no of individuals of all species.

\section{RESULTS}

The Kedarnath valley is very rich in terms of forest resources. Kedarnath valley is a highly elevated alpine meadow (bugyal) with a rich diversity of herbs, shrubs, and trees. Pine forest is common in mid-altitude, while in the upper reaches, temperate conifers forest, mainly Oak, Rhododendron, Devadar, Kafal are abundant. Many plant species of fodders, medicinal and fruit-bearing plants are common in this Valley. This study on the forest resources was carried out in nine sites of Kedarnath valley (Table 1).

The Kedarnath valley is blessed with the Himalayan Dry Temperate Forests, Dry Temperate Coniferous Forest and West Himalayan Birch/Fir Forests, Sub-Alpine Pasture, Himalayan Chir-Pine Forest, Himalayan Moist Temperate Forest, West Himalayan Sub-Alpine Birch/Fire Forest, and Alpine Forest.

The Forest cover of the study area has been presented in Table 2, and Karokhi has the largest forest cover area wise followed by Sari, Ransi, Ukhimath, Kabiltha, and Tungnath and Barasu have the lowest forest cover (Revenue report of the Village, Tehsil Ukhimath, R-57, 2016-17).

\section{PLANT BIODIVERSITY}

The terrestrial ecological survey for various aspects of the Kedarnath valley was conducted for a period of three years (2015 to 2018). The altitude in the villages of Kedarnath valley ranged from $864 \mathrm{~m}$ to $4,260 \mathrm{~m}$ asl. The major forest type of the valley was a mountain forest. A total number of 221 plant species were collected during the present study in the

\begin{tabular}{|c|c|c|c|c|}
\hline Study Site & Location & Latitude & Longitude & Altitude (m above m.s.1.) \\
\hline $\mathrm{S}_{1}$ & Chandrapuri & $30^{\circ} 25^{\prime} 29.72^{\prime \prime} \mathrm{N}$ & $79^{\circ} 04^{\prime} 17.68^{\prime \prime} \mathrm{E}$ & 864 \\
\hline $\mathrm{S}_{2}$ & Kalimath & $30^{\circ} 33^{\prime} 43.66^{\prime \prime} \mathrm{N}$ & $79^{\circ} 05^{\prime} 03.29^{\prime \prime} \mathrm{E}$ & 1,251 \\
\hline $\mathrm{S}_{3}$ & Gaundar & $30^{\circ} 36^{\prime} 09.76^{\prime \prime} \mathrm{N}$ & $79^{\circ} 10^{\prime} 47.29^{\prime \prime} \mathrm{E}$ & 1,653 \\
\hline $\mathrm{S}_{4}$ & Tarsali & $30^{\circ} 35^{\prime} 07.94^{\prime \prime} \mathrm{N}$ & 7901'16.97’"E & 1,805 \\
\hline $\mathrm{S}_{5}$ & Sari & $30^{\circ} 31^{\prime} 03.75^{\prime \prime} \mathrm{N}$ & $79^{\circ} 08^{\prime} 06.71^{\prime \prime} \mathrm{E}$ & 2,015 \\
\hline $\mathrm{S}_{6}$ & Gaurikund & $30^{\circ} 39^{\prime} 13.42^{\prime \prime} \mathrm{N}$ & $79^{\circ} 01 ’ 26.82{ }^{\prime \prime} \mathrm{E}$ & 2,156 \\
\hline $\mathrm{S}_{7}$ & Trijuginarayan & $30^{\circ} 38^{\prime} 25.55^{\prime \prime} \mathrm{N}$ & $78^{\circ} 58^{\prime} 30.01^{\prime \prime} \mathrm{E}$ & 2,246 \\
\hline $\mathrm{S}_{8}$ & Kedarnath & $30^{\circ} 44^{\prime} 07.38^{\prime \prime} \mathrm{N}$ & $79^{\circ} 04^{\prime} 00.57^{\prime \prime} \mathrm{E}$ & 3,560 \\
\hline $\mathrm{S}_{9}$ & Tungnath & $30^{\circ} 29^{\prime} 17.54^{\prime \prime} \mathrm{N}$ & $79^{\circ} 12^{\prime} 59.84^{\prime \prime} \mathrm{E}$ & 3,660 \\
\hline
\end{tabular}
Kedarnath valley. Plant diversity in the valley encompasses 49 tree species, 28 shrub species, and 144 herb species. An

Table 1: Study sites, their location, geographical coordinates, and elevations of the study area. 
inventory of plant species, their local names, family, and ethnobotanical uses have been presented in Table 3.

\section{Study Site $S_{1}$}

The study site $S_{1}$ was Chandrapuri village at the left bank of Mandakini River (864 m above m.s.l). This site has some scattered trees with few shrubs and plenty of herbs. The density, frequency, abundance, and Importance Value Index (IVI) of the trees, shrubs, and herbs at $\mathrm{S}_{1}$ have been presented in Table 4 and Table 5. Ecological analysis revealed the dominant tree species were Grewia optiva (IVI: 20.700), Banhinia variegata (IVI: 19.286), Pinus roxburghii (IVI: 16.921), and Toona ciliata (IVI: 16.303) at $S_{1}$. The dominant shrub species were Girardnia diversifolia (IVI: 30.774), Adhaoda zeylanica (IVI: 27.831), Lantana camara (IVI: 27.631), and Urtica dioica (IVI: 23.440). The dominant herb species were Galinsoga parviflora (IVI: 14.549), Euphurbia chamaesyce (IVI: 12.127), Reinwardtia indica (IVI: 11.902), and Ganatanthus pumilus (IVI: 11.798).

\section{Study Site $S_{2}$}

The study site $\mathrm{S}_{2}$ was Kalimath (1,251 $\mathrm{m}$ asl.) at the right bank of the Kali Ganga and left bank of the Mandakini River. The density, frequency, abundance, and Importance Value Index (IVI) of the trees, shrubs, and herbs have been presented in Table 4 and Table 5. Ecological analysis revealed the dominant tree species were Quercus liucotrichophora (IVI: 26.805), Alnus nepalensis (IVI: 24.373), Pyrus pashia (IVI: 20.456), and Pinus roxburghii (IVI: 17.741). However, the dominant shrub species were Solanum viarum (IVI: 31.703), Girardnia diversifolia (IVI: 28.478), and Berberis aristata (IVI: 24.146). The dominant herb species were Pilea umbrosa (IVI: 10.690), Laportea ovalifolia (IVI: 9.412) and Eulaliopsis binata (IVI: 9.311).

\section{Study Site $S_{3}$}

The study site $S_{3}(1,653 \mathrm{~m}$ asl.) was the Gaundar village at the right bank of the Madmaheswar Ganga. The density, frequency, abundance, and importance value index (IVI) of the trees, shrubs, and herbs have been presented in Table 4 and Table 5. Ecological analysis revealed the dominant tree species were Quercus liucotrichophora (IVI: 40.799), Alnus nepalensis (IVI: 30.639), and Myrica esculenta (IVI: 24.402). However, the dominant shrub species were Sarcococca saligna (IVI: 30.712), Adhatoda vasica (IVI: 30.402),

Table 2: Forest cover of Ukhiamth Tehsil in 2016-17 (Area in ha).

\begin{tabular}{|c|c|c|c|c|}
\hline S.No. & Name of villages & Altitude (m. above m.s.l.) & Geographical area (ha) & Forest Cover (ha) \\
\hline & Chandrapuri & 864 & 20.157 & 4.655 \\
\hline & Bhiri & 972 & 63.282 & 13.419 \\
\hline & Kalimath & 1,251 & 98.389 & 39.329 \\
\hline & Narayankoti & 1,396 & 29.408 & 9.876 \\
\hline & Ukhimath & 1,402 & 214.977 & 85.989 \\
\hline & Kabiltha & 1,408 & 49.105 & 22.404 \\
\hline & Guptakashi & 1,455 & 195.875 & 80.207 \\
\hline & Karokhi & 1,634 & 386.831 & 304.698 \\
\hline & Gaundar & 1,653 & 60.215 & 7.966 \\
\hline & Sersi & 1,686 & 85.96 & 16.86 \\
\hline & Barasu & 1,664 & 129.003 & 0.09 \\
\hline & Tarsali & 1,805 & 25.71 & 6.04 \\
\hline & Ransi & 1,974 & 253.634 & 118.833 \\
\hline & Sari & 2,015 & 286 & 254.702 \\
\hline & Gaurikund & 2,156 & 55.119 & 25.8 \\
\hline & Trijuginarayan & 2,246 & 419.426 & 29.66 \\
\hline & Tausi & 2,325 & 50.044 & 2.64 \\
\hline & Chopta & 2,862 & 3.62 & 2.845 \\
\hline & Kedarnath & 3,568 & 14.36 & - \\
\hline & Tungnath & 3,660 & 1.636 & 1.045 \\
\hline
\end{tabular}

Sources: Revenue Report of villages, Tehsil Ukhimath, R-57, 2016-17 
and Girardnia diversifolia (IVI: 28.320). The dominant herb species were Bidens pilosa (IVI: 14.015), Agrimonia pilusa (IVI: 12.262), and Euphurbia chamaesyce (IVI: 11.681).

\section{Study Site $\mathbf{S}_{4}$}

The study site $\mathrm{S}_{4}(1,805 \mathrm{~m}$ a.s.l) was the Tarsali village, located at the right bank of the Mandakini River. The density, frequency, abundance, and Importance Value Index (IVI) of the trees, shrubs, and herbs have been presented in Table 4 and Table 5. Ecological analysis revealed the dominant tree species were Quercus liucotrichophora (IVI: 43.294), Rhododendron arboreum (IVI: 28.921), and Neolitsea sericea (IVI: 19.164). However, the dominant shrub species were Sarcococca saligna (IVI: 51.337), Girardnia diversifolia

Table 3: Inventory of plant species, their local names, and ethnobotanical uses in the study area of Kedarnath valley

\begin{tabular}{|c|c|c|c|c|}
\hline S.No. & Name of Species & Local Name & Family & Ethnobotanical Uses \\
\hline \multicolumn{5}{|l|}{ Trees } \\
\hline 1. & Abies spectabilis (D.Don) Spach & & Pinaceae & Timber, Fuel \\
\hline 2. & Aesculus indica (Wall.ex Camb.) Hook & Pangar & Sapindaceae & Medicinal, Wild edible, Fuel \\
\hline 3. & Alnus nepalensis D.Don & Utis & Betulaceae & Timber, Fuel \\
\hline 4. & Banhinia variegata $\mathrm{L}$. & Kachnar & Caesalpinaceae & Medicinal, Wild edible, Fuel \\
\hline 5. & Betula alnoides Buch. -Ham.ex D.Don & Saur, sore & Betulaceae & Timber, Fuel, Fodder \\
\hline 6. & Cedrus deodara (Roxb.) G.Don & Deodara & Pinaceae & Timber, Fuel \\
\hline 7. & Celtis australis L. & Khadik & Cannabaceae & Fodder, Fuel \\
\hline 8. & Cinnamomum Spp. Schaeff. & & Lauraceae & Fodder, edible, Fuel \\
\hline 9. & Cotoneaster affinis Lindl. & Ruins & Rosaceae & Fuel, Agriculture tool \\
\hline 10. & Debregeasia longifolia (Burm.F.) Wedd. & Syanru & Urticaceae & Fodder, edible, Fuel \\
\hline 11. & Emblica officinalis Gaertn. & Aonla & Euphorbiaceae & Medicinal, edible, Fuel \\
\hline 12. & Ficus auriculata Lour. & Timla & Moraceae & Fodder, fruit edible \\
\hline 13. & Ficus palmata Forsk. & Bedu & Moraceae & Fodder, fruit edible, medicinal \\
\hline 14. & Ficus religiosa $\mathrm{L}$. & Peepal & Moraceae & Medicinal, Fuel \\
\hline 15. & Ficus semicordata Bunch.-ham.ex J. E.Smith & Khannu & Moraceae & Medicinal, Wild edible, Fuel \\
\hline 16. & Fraxinus americana $\mathrm{L}$. & Anga & Oleaceae & Fuel, Timber \\
\hline 17. & Grewia optiva Drummond ex Burrt & Bhimal & Tiliaceae & Fuel, Fodder \\
\hline 18. & Hippophae salicifolia D.Don & & Elaeagnaceae & Medicinal, Wild edible, Fuel \\
\hline 19. & Holmskiodia sanguinea & Khagsoo & Verbinaceae & Fuel, Fodder \\
\hline 20. & Juglans regia $\mathrm{L}$. & Akhrot & Juglandaceae & Medicinal, Wild edible, Fish poison, Fuel \\
\hline 21. & Lyonia ovalifolia (Wall.) Prude & Anyar & Ericaceae & Fuel, Fish Poison, Medicinal, Fodder \\
\hline 22. & Mangifera indica $\mathrm{L}$. & Aam & Anacardiaceae & Fruit edible, woodwork \\
\hline 23. & Morus alba $\mathrm{L}$. & Sahtoot & Moraceae & Fruit edible woodwork, sericulture \\
\hline 24. & Myrica esculenta Buch. -Ham.ex D.Don & Kafal & Myricaceae & Medicinal, Wild edible, Fish poison, Fuel \\
\hline 25. & Neolitesa serobiculata (Meisn.) Gamble & Gadmweda & Lauraceae & Fuel \\
\hline 26. & Neolitsea sericea (Blume) Koidz. & (Mweda, chirad) & Lauraceae & Fuel \\
\hline 27. & Neolitsea Spp. (Bent. \& Hook.F.) Merr. & Lampatiya & Lauraceae & Fuel, Timber \\
\hline 28. & Phoenix humilis Royle. & Khajoor & Arecaceae & Medicinal, Wild edible, Fuel \\
\hline 29. & Pinus roxburghii Sarjent & Kulain & Pinaceae & $\begin{array}{l}\text { Wood for construction, resin, medicinal, } \\
\text { timber }\end{array}$ \\
\hline 30. & Prunus cerasoides D.Don & Panya & Rosaceae & Medicinal, Wild edible, Fuel, Timber \\
\hline 31. & Prunus cornuta (Wall. ex Royle) & $\begin{array}{l}\text { Himalayan bird } \\
\text { cherry, padus }\end{array}$ & Rosaceae & Medicinal, Wild edible, Fuel, Timber \\
\hline
\end{tabular}




\begin{tabular}{|c|c|c|c|c|}
\hline S.No. & Name of Species & Local Name & Family & Ethnobotanical Uses \\
\hline 32. & Pyrus pashia Buch. -Ham.ex D.Don & Mol & Rosaceae & Fodder, Fuel, Medicinal, Wild edible \\
\hline 33. & Quercus floribunda Lindley.ex Rehder & Moru & Fabuceae & Fodder, Fuel \\
\hline 34. & Quercus liucotrichophora A.Camus & Banj & Fagaceae & Fodder, Fuel \\
\hline 35. & Quercus semecarpifolia $\mathrm{Sm}$. & Karsu & Fagaceae & Fodder, Fuel \\
\hline 36. & Quercus Spp. L. & Harinj, Green oke & Fagaceae & Fodder, Fuel \\
\hline 37. & Rhododendron arboreum $\mathrm{Sm}$. & Burans & Ericaceae & Medicinal, Wild edible, Fuel, Timber \\
\hline 38. & Rhododendron barbatum Wallich ex G. Don & & Ericaceae & Medicinal, Wild edible, Fuel, Timber \\
\hline 39. & Rhus sandwicensis A.Gray & Titret & Anacardiaceae & Fuel, Fodder \\
\hline 40. & Rosa sericea Lindl. & & Rosaceae & Medicinal, Fuel \\
\hline 41. & Sapindus mukorossi Gaertner & Reetha & Sapindaceae & Medicinal, Fuel, Timber \\
\hline 42. & Symplocos panniculata (Thunb.) Miq & Lodha & Symplocaceae & Fodder. Fuel \\
\hline 43. & Syzygium cumiini (L.) Skeels & Jamun & Myrataceae & Medicinal, Wild edible, Fuel, Timber \\
\hline 44. & Taxcus baccata $\mathrm{L}$. & Thuner & Taxaceae & Medicinal, Timber, fuel \\
\hline 45. & Taxus wallichiana Zucc. & & Taxaceae & Medicinal, Timber, fuel \\
\hline 46. & Toona ciliata Roem. & Toon & Meliaceae & Timber and wood work, social forestry \\
\hline 47. & Ulmus wallichiana Planch. & Pamani,mairu & Urticaceae & Fodder, Fuel \\
\hline 48. & Viburmum mullaha Buch. -Ham.ex D.Don & Malyo & Caprifoliaceae & Fodder, Fuel, Medicinal, Wild edible \\
\hline 49. & Zanthoxylum armatum DC & Timaru & Rutaceae & Fodder, Fuel, Medicinal \\
\hline \multicolumn{5}{|c|}{ Shrubs } \\
\hline 1. & $\begin{array}{l}\text { Ageratina adenophora (Spreng.) King \& } \\
\text { H.Rob. }\end{array}$ & Basinga & Acanthaceae & Medicinal \\
\hline 2. & Arismia tortosum & & & Medicinal \\
\hline 3. & Berberis aristata Roxb.ex.DC. & Kirmor & Berberidaceae & Wild edible, Medicinal, Fuel \\
\hline 4. & Berberis jaeschkeana DC. & & Berberidaceae & Wild edible, Medicinal, Fuel \\
\hline 5. & Boehmeria platyphylla D.Don & Khagsa & Urticaceae & Fodder, Fuel \\
\hline 6. & Caesalipinia decapetala (Roth) Alston & Kingari,kunju & Caesalpiniaceae & Fodder, Medicinal, Fuel \\
\hline 7. & Cannabis sativa Linn. & Bhang & Cannabinaceae & $\begin{array}{l}\text { Bark fibers for ropes, sacs, and rough } \\
\text { clothes, seeds as condiment, intoxicating }\end{array}$ \\
\hline 8. & Cotoneaster microphyllus Wall. ex Lindl. & & Malaceae & Wild edible, Medicinal, Fuel \\
\hline 9. & Desmodium concimum DC. & Sakina & Fabaceae & Fodder, Fuel \\
\hline 10. & Desmodium elegans DC. & Chamlai & $\begin{array}{l}\text { Fabaceae, Papilion- } \\
\text { aceae }\end{array}$ & Fodder, Fuel \\
\hline 11. & Echinops cornigenus & Kandara & Asterceae & Medicinal, Edible \\
\hline 12. & Elueagnus parvifolia Wall.ex Royal & Giwain & Elueagnaceae & Wild edible, Medicinal, Fuel \\
\hline 13. & Girardnia diversifolia (Link) Friis & Jhir kandali & Urticaceae & Fodder, Medicinal \\
\hline 14. & Lantana camara $\mathrm{L}$. & Gajar ghass & Verbenaceae & Fuel, furniture, Medicinal, Weed \\
\hline 15. & Lonicera $x$ bella Zabel & Ghugti & Carprifoliaceae & Fuel \\
\hline 16. & Prisepia utilis Royle & Bhenkul & Rosaceae & Medicinal, Fuel \\
\hline 17. & Pyracantha crenulata (D.Don) M.Roem. & Ghingaru & Rosaceae & Soil binder, fruit edible, Medicinal, Fuel \\
\hline 18. & Rhododendron barbatum Wallich ex G. Don & & Ericaceae & Medicinal, Wild edible, Fuel, Timber \\
\hline 19. & Rhododendron campanulatum D.Don & Burans & Ericaceae & Medicinal, Wild edible, Fuel, Timber \\
\hline 20. & Rosa spp. L. & & Rosaceae & Medicinal, Fuel \\
\hline
\end{tabular}




\begin{tabular}{|c|c|c|c|c|}
\hline S.No. & Name of Species & Local Name & Family & Ethnobotanical Uses \\
\hline 21. & Rubus ellipticus $\mathrm{Sm}$. & Hinsalu & Rosaceae & Fruit edible \\
\hline 22. & Rubus niveus Thunb. & Kali hisar & Rosaceae & Fruit edible \\
\hline 23. & Sarcococca saligna (D.Don) & Geru, paliyala & Buxaceae & Medicinal, Fuel \\
\hline 24. & $\begin{array}{l}\text { Sinarumdinaria anceps (Mittf.) Chao \& Ren- } \\
\text { voize.Sqn. }\end{array}$ & Ringal & Poaceae & Fuel \\
\hline 25. & Smilax aspera L. & Kukardara & Smilacaceae & Medicinal \\
\hline 26. & Solanum viarum Dunal & & Solanaceae & Medicinal \\
\hline 27. & Urtica dioica $\mathrm{L}$. & Kandali & Urticaceae & Edible, Medicinal \\
\hline 28. & Viburnum spp. L. & & Adoxaceae & Medicinal \\
\hline \multicolumn{5}{|l|}{ Herbs } \\
\hline 1. & Abies pindrow (Royle ex D.Don) Royle & & Pinaceae & Medicinal, Edible \\
\hline 2. & Abrus precatorius L. & Ratti & Fabaceae & Medicinal \\
\hline 3. & Acomastylis elata (Wall.ex G.Don) F.Bolle & & Rosaceae & Medicinal \\
\hline 4. & Agrimonia pilusa Ledebour & Lisukuri & Rosaceae & Fodder \\
\hline 5. & Ampelocissus latifolia Planch. & & Araliaceae & Fodder, Medicinal \\
\hline 6. & Anaphalis beddomei Hook.F. & & Asteraceae & Medicinal \\
\hline 7. & Anaphalis contorta (D.Don) Hook.f. & & Asteraceae & Medicinal \\
\hline 8. & Anaphalis royleana DC & & Asteraceae & Medicinal \\
\hline 9. & Anaphalis spp. DC. & & Asteraceae & Medicinal \\
\hline 10. & Anaphalis spp.DC. & & Asteraceae & Medicinal \\
\hline 11. & Androsace lanuginosa Wall. & & Primulaceae & Medicinal \\
\hline 12. & Anemone obtusiloba D.Don, Prode. Fl. & & Ranunculaceae & Medicinal \\
\hline 13. & Anemone patens $\mathrm{L}$. & & Rosaceae & Medicinal \\
\hline 14. & Anemone spp L. & & Ranunculaceae & Medicinal \\
\hline 15. & Anemone vitifolia Buch.-Ham. ex DC. & & Ranuculaceae & Medicinal \\
\hline 16. & Animone obtusiloba D.Don & & Renunculaceae & Medicinal \\
\hline 17. & Arisaema flavam (Foessk.) Schott & & Araceae & Medicinal \\
\hline 18. & Arisaema intermedium BL. & Akash laguli & Convolvulaceae & Medicinal \\
\hline 19. & Arisaemia tortosum (Wall.) Schott & & Araceae & Medicinal \\
\hline 20. & Arisuema totuosum (Wall.) Schot & Bell type & Vitaceae & Medicinal \\
\hline 21. & Aster spp. L. & & Asteraceae & Medicinal \\
\hline 22. & Bauhinia vahlii Wight \& Arn. & Bagmungari & Araceae & Medicinal \\
\hline 23. & Bergenia ciliata (Haworth) Stern. & Silpara & Saxifragaceae & Medicinal \\
\hline 24. & Bidens pilosa $\mathrm{L}$. & Kumar & Astoraceae & Medicinal, Fodder \\
\hline 25. & Bistorta macrophylla (D.Don) Sojak & & Polygonaceae & Medicinal \\
\hline 26. & Bistorta vaccinifolia Wall. ex Meisn.) & & Polygonaceae & Medicinal \\
\hline 27. & Boehmeria grandis(Hook. \& Arn.) A. Heller & Foortya & Urticaceae & Fodder \\
\hline 28. & Boehmeria nivea (L.) Gaudich. & & Urticaceae & Medicinal \\
\hline 29. & Boenninghausenia albiflora & Upniya ghass & Rutaceae & Fodder, Medicinal \\
\hline 30. & Bupleurum fruticosum $\mathrm{L}$. & & Apiaceae & Fodder \\
\hline 31. & Carax hirta L. & & Cyperaceae & Fodder \\
\hline 32. & Carex spp. L. & & Cyperaceae & Fodder \\
\hline
\end{tabular}




\begin{tabular}{|c|c|c|c|c|}
\hline S.No. & Name of Species & Local Name & Family & Ethnobotanical Uses \\
\hline 33. & Centella asiatica $\mathrm{L}$. & Brahmi & Apiaceae & Medicinal \\
\hline 34. & Chenopodium album $\mathrm{L}$. & Bathua & Chenopodiaceae & Edible, Fodder, Medicinal \\
\hline 35. & Citrullus colocynthis (L.) Schrader & & Rutaceae & Medicinal \\
\hline 36. & Clematis montana Buch.-Ham. ex DC. & & Ranunculaceae & Medicinal \\
\hline 37. & Corydalis cornuta Royal & & Papaveraceae & Medicinal \\
\hline 38. & Crepidium acminatum (D.Don) Szlach. & Jeevak & Orchidaceae & Medicinal \\
\hline 39. & Cuscuta reflexa Roxb. & Dudhi & Euphorbiaceae & Medicinal \\
\hline 40. & Cyananthus lobatus Wall. ex Benth & & Campanulaceae & Medicinal \\
\hline 41. & Cyathula tomentosa (Roth) Moq. & Lichkura & Amarnathaceae & Fodder, Medicinal \\
\hline 42. & Cymbopogon citratus (DC.) Stapf & Lemongrass & Poaceae & Fodder, Medicinal \\
\hline 43. & Cynodon dactylon (L.) Pers. & Doob & Poaceae & Medicinal, Fodder \\
\hline 44. & Cynoglossum zeylanicum $\mathrm{L}$. & & Boraginaceae & Medicinal \\
\hline 45. & Cyperus odoratus $\mathrm{L}$. & Ghass & Cyperaceae, Poaceae & Fodder \\
\hline 46. & Danthonia cachmiriana $\mathrm{L}$. & & Poaceae & Fodder \\
\hline 47. & Danthonia spp. DC. & & Poaceae & Fodder \\
\hline 48. & Daphne papyracea Wall. & Kandara & Asteraceae & Fodder \\
\hline 49. & Digitaria ciliaris (Retz.) Koeler & Menaru & Poaceae & Fodder \\
\hline 50. & Dioscorea belophylla (Prain) Haines Syn. & Tedu & Deoscoreaceae & Edible, Medicinal \\
\hline 51. & Dioscorea Spp. L. & & Dioscoreaceae & Edible \\
\hline 52. & Diplazium caudatum (Cav.) Jermy & Farn & Athyriacae & Medicinal \\
\hline 53. & Diplazium esculentum (Retz.) SW. & Lingra & $\begin{array}{l}\text { Dryopteridaceae, } \\
\text { Athyriaceae }\end{array}$ & Edible, Medicinal \\
\hline 54. & Diplazium melanochlamys (Hook.) T.Moore & Una, fern & Athyriacae & Fodder, Medicinal \\
\hline 55. & Diplazium splendens Ching & Meen & Araceae & Medicinal \\
\hline 56. & Diplazium spp. & Bis lingara & $\begin{array}{l}\text { Dryopteridaceae, } \\
\text { Athyriaceae }\end{array}$ & Medicinal \\
\hline 57. & Dryopteris filixmas (L.) Schott & Fern & Dryopteridaceae & Medicinal, Fodder \\
\hline 58. & Dryopteris filix -mas & & Dryopteridaceae & Medicinal \\
\hline 59. & Dubyaea hispida (D.Don) DC. & & Asteraceae & Medicinal \\
\hline 60. & Duchesnea indica (Andrcos) Th.Wolf & Bhina kafal & Rosaceae & Edible, Medicinal \\
\hline 61. & Echinops cornigenus Roxb. & Meda & Asparagaceae & Medicinal \\
\hline 62. & Epilobium hirsutum L. & & Onagraceae & Medicinal \\
\hline 63. & Eulaliopsis binata (Retz.) C.E. Hubb. & Ban pindalu & Araceae & Medicinal. Edible \\
\hline 64. & Euphorbia spp.L. & & Euphorbiaceae & Medicinal \\
\hline 65. & Euphurbia chamaesyce L. & Ban-haldi & Zingiberaceae & Fodder, Medicinal \\
\hline 66. & Evolvulus alsinoides (L.) L. & Sankpushpi & Convolvulaceae & Medicinal \\
\hline 67. & Fagopyrum esculentum (L.) Moench. & Konlya, ougal & Polygonaceae & Fodder, Medicinal, Edible \\
\hline 68. & Festuca spp. L. & Grass & Poaceae & Fodder \\
\hline 69. & Fragaria rubicola $\mathrm{L}$. & & Rosaceae & Medicinal \\
\hline 70. & Fumaria indica (haussk.) Pugsl. & Pit-papra & Liliaceae & Medicinal \\
\hline 71. & Galinsoga parviflora Cav. & Khor type & Poaceae & Fodder \\
\hline 72. & Ganatanthus pumilus (D.Don) Engl. \& Krause & Badelu grass & Asteraceae & Fodder, Medicinal \\
\hline
\end{tabular}




\begin{tabular}{|c|c|c|c|c|}
\hline S.No. & Name of Species & Local Name & Family & Ethnobotanical Uses \\
\hline 73. & Gaultheria trichophylla Royle & & Ericaceae & Medicinal \\
\hline 74. & Geum elatum Wall. Ex G.Don & & Rosaceae & Medicinal \\
\hline 75. & Gleichenia spp. Sm. & & Gleicheniaceae & Medicinal \\
\hline 76. & Hedra nepalensis K.Koch & Ivi & Polygonaceae & Medicinal \\
\hline 77. & Hedychium spicatum Buch.-Ham. & Phiyunli & Liliaceae & Medicinal \\
\hline 78. & Heracleum maximum Bartr. & & Asteraceae & Medicinal \\
\hline 79. & Impatiens scabrida DC. & & Balsaminaceae & Medicinal \\
\hline 80. & Impatiens sulcata Wall. & Majuro & Balsaminaceae & Medicinal \\
\hline 81. & Ischaemum rugosum Salisb. & & Poaceae & Medicinal, Fodder \\
\hline 82. & Juniperus squamata Buch.-Ham. ex D.Don & & Cupressaceae & Medicinal \\
\hline 83. & Laportea ovalifolia Schum. (Thonn.) Chew & Malcharu & Nasselxaxter & Fodder \\
\hline 84. & Lathyrus spp. L. & Kurfalya & fabaceae & Edible, Fodder, Medicinal \\
\hline 85. & Lonicera obovata Royle & & Carprifolvaxter & Medicinal \\
\hline 86. & Oplismenus hirtellus (L.) P.Beauv. & $\begin{array}{l}\text { Menaru, basket } \\
\text { grass }\end{array}$ & Poaceae & Fodder \\
\hline 87. & Oxalis corniculata $\mathrm{L}$. & Bhilmori & Oxalidaceae & Edible, Fodder, Medicinal \\
\hline 88. & Oxora coccinea $\mathrm{L}$. & & Rubiaceae & Medicinal \\
\hline 89. & Oxyria digyna (L) Hill & & Polygonaceae & Medicinal \\
\hline 90. & Paeonia emodi Royal & Dhanduru & Paeoniaceae & Edible, Medicinal \\
\hline 91. & Parthenocissus semicordata (Wall) Planch. & & Vitaceae & Medicinal \\
\hline 92. & $\begin{array}{l}\text { Persicaria amplexicaulis (D. Don) Ronse } \\
\text { Decraene }\end{array}$ & & Polygonaceae & Medicinal \\
\hline 93. & Pilea umbrosa Wall.ex B1. & Chaolu & Urticaceae & Fodder \\
\hline 94. & Plantago brachyphylla Edgew. ex Decne & & Plantaginaceae & Medicinal \\
\hline 95. & Plantago deprassa Willd. & Luhurya, symlya & Planttaginaceae & Medicinal \\
\hline 96. & Plantago spp. L. & & Plantaginaceae & Medicinal \\
\hline 97. & Podophyllum hexandrum Royle & Ban kakdi & Podophyllaceae & Edible, Medicinal, Fodder \\
\hline 98. & Polygonatum verticillatam (L.) All. & Malu & Caesalpiniaceae & Medicinal \\
\hline 99. & $\begin{array}{l}\text { Polygonum capitatum (Buch.-Ham. Ex D.Don) } \\
\text { H.Gross }\end{array}$ & & Renunculaceae & Medicinal \\
\hline 100. & Polygonum filicaule Wall. ex Meissn & & Polygonaceae & Medicinal \\
\hline 101. & Polygonum spp. L. & & Polygonaceae & Medicinal \\
\hline 102. & Polygonum polystachyum Wall. ex Meissn & & Polygonaceae & Medicinal \\
\hline 103. & Poteatilla spp. L. & & Rosaceae & Medicinal \\
\hline 104. & Potentilla atrosangunea G.LOOD.ex D.Don & & Rosaceae & Medicinal \\
\hline 105. & Potentilla fulgens L. & & Rosaceae & Medicinal \\
\hline 106. & Potentilla fulgens Wall. Ex HK.F. & Bajaradanti & Rosaceae & Medicinal \\
\hline 107. & Potentilla spp. L. & & Rosaceae & Medicinal \\
\hline 108. & Potentilla polyphylla Wall. ex Lehm. & & Rosaceae & Medicinal \\
\hline 109. & Potentilla polyphylla Wall. ex Lehm & & Rosaceae & Medicinal \\
\hline 110. & Primula spp L. & & Primulaceae & Medicinal \\
\hline 111. & Prunella vulgaris $\mathrm{L}$. & & Lamiaceae & Medicinal \\
\hline
\end{tabular}




\begin{tabular}{|c|c|c|c|c|}
\hline S.No. & Name of Species & Local Name & Family & Ethnobotanical Uses \\
\hline 112. & Ranunculus hirtellus Royle & & Ranunculaceae & Medicinal \\
\hline 113. & Ranunculus repens $\mathrm{L}$. & & Apiaceae & Medicinal \\
\hline 114. & Reinwardtia indica Dum. & Bugla & Asterceae & Medicinal \\
\hline 115. & Rhododendron anthopogon D.Don & Burans & Ericaceae & Medicinal, Wild edible, Fuel, Timber \\
\hline 116. & Rubia manjith Roxb.ex Fleming & $\begin{array}{l}\text { Lichkuri, indian } \\
\text { madder }\end{array}$ & Rubiaceaae & Medicinal, Fodder \\
\hline 117. & Rumex hastatus D.Don & Almora & Polygonaceae & Edible, Fodder, Medicinal \\
\hline 118. & Rumex nepalensis Speeng. & & Polygonaceae & Medicinal \\
\hline 119. & Saccharum officinarum $\mathrm{L}$. & & Poaceae & Medicinal \\
\hline 120. & Salix lindleyana Wallich ex Ander. & & Salicaceae & Medicinal \\
\hline 121. & Salix spp L. & & Salicaceae & Medicinal \\
\hline 122. & Salvia nubicola Wall. ex Sweet & & Lamiaceae & Medicinal \\
\hline 123. & Saxifraga parnassifolia D.Don & & Saxifragaceae & Medicinal \\
\hline 124. & Scrophularia californica Cham. \& Schldl. & & Scrophulariaceae & Medicinal \\
\hline 125. & Sedum spp L. & & Crassulaceae & Medicinal \\
\hline 126. & Selenium cuneifokiaa DC. & & Asteraceae & Medicinal \\
\hline 127. & Senecio spp $\mathrm{L}$. & & Asteraceae & Medicinal \\
\hline 128. & Sibbaldia cuneata Hornem.ex Kuntze & & Rosaceae & Medicinal \\
\hline 129. & Smilax aspera $\mathrm{L}$. & & Smilacaceae & Medicinal \\
\hline 130. & Solanum nigrum $\mathrm{L}$. & & Solanaceae & Fodder, Medicinal \\
\hline 131. & Sweritia chirayia (Roxb. Ex Fleming) Karsten & Chiraita & Gentianaceae & Medicinal \\
\hline 132. & Tanacetum longifolium Wall. ex DC. & & Asteraceae & Medicinal \\
\hline 133. & Taraxacum officinale (L.) Weber ex F.H. Wigg & & Asteraceae & Medicinal \\
\hline 134. & Taraxacum officinale (L.) Weber ex F.H.Wigg & & Asteraceae & Medicinal \\
\hline 135. & Tetratigma spp. Merr.\& Chun & Bell-type & Vitaceae & Edible, Medicinal \\
\hline 136. & Tetratigma pubinerve & & Thymelaeaceae & Medicinal \\
\hline 137. & Tetratigma serrulatum (Roxb.) Planch. & & Vitaceae & Medicinal \\
\hline 138. & Teucrium quadnfarium Buch -Ham. & & Lamiaceae & Medicinal \\
\hline 139. & Trichosanthes tricuspidata Lour. & Ilaru & Cucurbiaceae & Medicinal \\
\hline 140. & Trychidium royle & & & Medicinal \\
\hline 141. & Urtica dioica $\mathrm{L}$. & & Urticaceae & Fodder \\
\hline 142. & Valeriana herdwikaii Wall. & & Caprifoliaceae & Medicinal \\
\hline 143. & Viburnum grandiflorum Wall. & & Adoxaceae & Medicinal \\
\hline 144. & Viola biflora $\mathrm{L}$. & Bana-ksha & Violaceae & Medicinal \\
\hline
\end{tabular}

(IVI: 35.807), and Berberis aristata (IVI: 22.769). The dominant herb species were Oplismenus hirtellus (IVI: 21.957), Cyperus odoratus (IVI: 18.375), and Ischaemum rugosum (IVI: 15.377).

\section{Study Site $S_{5}$}

The study site $S_{5}(2,015 \mathrm{~m}$ a.s.l) was the Sari village, located at the left bank of the Mandakini River. The density, frequency, abundance, and Importance Value Index (IVI) of the trees, shrubs, and herbs have been presented in Table 4 and Table 5. Ecological analysis revealed the dominant tree species were Quercus liucotrichophora (IVI: 25.677), Alnus nepalensis (IVI: 21.965), and Aesculus indica (IVI: 21.701). However, the dominant shrub species were Girardnia diversifolia (IVI: 40.998), Sarcococca saligna (IVI: 27.752), and Urtica dioica (IVI: 25.216). The dominant herb species were Pilea umbrosa (IVI: 20.192), Cymbopogon citratus (IVI: 16.016), and Cymbopogon citratus (IVI: 16.016). 
Table 4: Dominance of tree, shrub, and herb species and Total Basal Area (TBA) of plant species in the study area of Kedarnath valley

\begin{tabular}{|llllll|}
\hline S.N. & Village Name & Dominance of tree species & Dominance of shrub species & \multicolumn{2}{l|}{$\begin{array}{l}\text { Dominance of herb tree } \\
\text { species }\end{array}$} \\
\hline 1. & Chandrapuri & Grewia optiva & Girardnia diversifolia & Galinsoga parviflora & Banhinia variegata \\
2. & Kalimath & Quercus liucotrichophora & Solanum viarum & Pilea umbrosa & Quercus leucotrichophora \\
3. & Gaundar & Quercus liucotrichophora & Sarcococca saligna & Bidens pilosa & Quercus leucotrichophora \\
4. & Tarsali & Quercus liucotrichophora & Sarcococca saligna & Oplismenus hirtellus & Quercus leucotrichophora \\
5. & Sari & Quercus liucotrichophora & Girardnia diversifolia & Pilea umbrosa & Quercus leucotrichophora \\
6. & Gaurikund & Quercus liucotrichophora & Echinops cornigenus & Oplismenus hirtellus & Quercus leucotrichophora \\
7. & Trijuginarayan & Quercus liucotrichophora & Sarcococca saligna & Agrimonia pilusa & Quercus leucotrichophora \\
8. & Kedarnath & Taxus wallichiana & Berberis jaeschkeana & Trychidium royle & Taxus wallichiana \\
9. & Tungnath & Abies spectabilis & Rhododendron campanulatum & Carax hirta & Rhododendron barbatum \\
\hline
\end{tabular}

\section{Study Site $S_{6}$}

The study site $\mathrm{S}_{6}(2,156 \mathrm{~m}$ a.s.l) was the Gaurikund village, located at the right bank of the Mandakini River. The density, frequency, abundance, and Importance Value Index (IVI) of the trees, shrubs, and herbs have been presented in Table 4 and Table 5. Ecological analysis revealed the dominant tree species were Quercus liucotrichophora (IVI: 38.35), Neolitsea sericea (IVI: 35.87), and Betula alnoides (IVI: 24.25). However, the dominant shrub species were Echinops cornigenus (IVI: 52.24), Girardnia diversifolia (IVI: 35.31), and Sarcococca saligna (IVI: 26.49). The dominant herb species were Oplismenus hirtellus (IVI: 18.55), Cymbopogon citratus (IVI: 17.25), and Diplazium esculentum (IVI: 16.59).

\section{Study Site $S_{7}$}

The study site S7 (2,246 m a.s.l) was the Trijuginarayan village, located at the right bank of the Mandakini River. The density, frequency, abundance, and Importance Value Index (IVI) of the trees, shrubs, and herbs have been presented in Table 4 and Table 5. Ecological analysis revealed the dominant tree species were Quercus liucotrichophora (IVI: 48.10), Rhododendron arboreum (IVI: 28.37), and Neolitsea sericea (IVI: 25.40). However, the dominant shrub species were Sarcococca saligna (IVI: 33.43), Girardnia diversifolia (IVI: 24.12), and Cannabis sativa (IVI: 18.25). The dominant herb species were Agrimonia pilusa (IVI: 22.48), Bidens pilosa (IVI: 14.48), and Diplazium esculentum (IVI: 14.27).

Table 5: Different ecological and diversity parameters in the study area of Kedarnath valley.

\begin{tabular}{|c|c|c|c|c|c|c|c|c|c|}
\hline Parameters & Chandrapuri & Kalimath & Gaundar & Tarsali & Sari & Gaurikund & Trijuginarayan & Kedarnath & Tungnath \\
\hline $\begin{array}{l}\text { Tree density } \\
\text { (ind. } 100 \mathrm{~m}^{-2} \text { ) }\end{array}$ & 38.4 & 100.7 & 37.9 & 81 & 46 & 54.3 & 49.4 & 6.8 & 15.5 \\
\hline $\begin{array}{l}\text { Shrub density } \\
\text { (ind } 25 \mathrm{~m}^{-2} \text { ) }\end{array}$ & 81.5 & 95 & 122.9 & 46.8 & 65 & 36.9 & 49.1 & 15 & 11.8 \\
\hline $\begin{array}{l}\text { Herb density } \\
\left(\text { ind. } m^{-2}\right)\end{array}$ & 200 & 323.4 & 248.7 & 202.8 & 198.9 & 197.6 & 125.5 & 229.7 & 147 \\
\hline TBC $\left(\mathrm{m}^{2} \mathrm{ha}^{-1}\right)$ & 21.828 & 57.364 & 20.417 & 33.606 & 34.086 & 43.704 & 38.28 & 21.475 & - \\
\hline Tree IVI & 300.001 & 299.995 & 300.002 & 299.989 & 300.004 & 299.83 & 299.99 & 300 & 299.98 \\
\hline Shrub IVI & 299.998 & 300.002 & 299.999 & 300.006 & 299.999 & 300.01 & 231.55 & 300.01 & 300 \\
\hline Herb IVI & 300.002 & 300.001 & 300.001 & 300 & 299.997 & 299.99 & 300.01 & 299.99 & 300.02 \\
\hline $\begin{array}{l}\text { Shannon Index } \\
\text { (Tree) }(\bar{H})\end{array}$ & 3.028 & 3.048 & 2.901 & 3.001 & 2.918 & 2.753 & 2.636 & 1.737 & - \\
\hline $\begin{array}{l}\text { Shannon Index } \\
\text { (Shrub) }(\bar{H})\end{array}$ & 2.788 & 2.696 & 2.629 & 2.492 & 2.594 & 2.404 & 2.047 & 0.192 & - \\
\hline $\begin{array}{l}\text { Shannon Index } \\
\text { ( Herb) }(\bar{H})\end{array}$ & 3.613 & 3.787 & 3.531 & 3.305 & 3.156 & 3.367 & 3.317 & 3.712 & 3.115 \\
\hline
\end{tabular}

IVI=Importance Value Index; TBA=Total Basal Area; $\bar{H}=$ Diversity Index 


\section{Study Site $\mathbf{S}_{\mathbf{8}}$}

The study site $\mathrm{S}_{8}(3,568 \mathrm{~m}$ a.s.l) was the Kedarnath, located at the right bank of the Mandakini River. The density, frequency, abundance, and Importance Value Index (IVI) of the trees, shrubs, and herbs have been presented in Table 4 and Table 5. Ecological analysis revealed the dominant herb species were Trychidium royle (IVI: 17.38), Danthonia spp. (IVI: 12.00) and Anaphalis spp. (IVI: 11.16). However, the dominant shrub species were Berberis jaeschkeana (IVI: 35.81), Rosa spp. (IVI: 21.38), and Arismia tortosum (IVI: 15.39). The dominant tree species were Taxus wallichiana (IVI: 66.82), Abies spectabilis (IVI: 63.44), and Rhododendron barbatum (IVI: 50.12).

\section{Study site $\mathbf{S}_{\mathbf{9}}$}

The study site $S_{9}$ (3,660 m a.s.l) was the Tungnath, located at the left bank of the Mandakini River. The density, frequency, abundance, and Importance Value Index (IVI) of the trees, shrubs, and herbs have been presented in Table 4 and Table 5. Ecological analysis revealed the dominant herb species were Carax hirta (IVI: 26.23), Potentilla fulgens (IVI: 20.98), and Rhododendron anthopogon (IVI: 19.27). However, the dominant shrub species were Rhododendron campanulatum. The dominant tree species were Abies spectabilis.

\section{Total basal area (TBA)}

In the Chandrapuri forest area, the total basal area was higher for Banhinia variegata $\left(1.978 \mathrm{~m}^{2} \mathrm{ha}^{-1}\right)$, possibly due to a higher density of trees (Table 4 and Table 5). In the Kalimath forest area, the total basal area was higher for Quercus liucotrichophora $\left(7.688 \mathrm{~m}^{2} \mathrm{ha}^{-1}\right)$, possibly due to a higher density of trees (Table 4 and Table 5). In the Gaundar forest area, the total basal area was higher for Quercus liucotrichophora $\left(4.864 \mathrm{~m}^{2} \mathrm{ha}^{-1}\right)$, possibly due to a higher density of trees (Table 4 and Table 5). In the Tarsali forest area, the total basal area was higher for Quercus liucotrichophora $(9.542$ $\mathrm{m}^{2} \mathrm{ha}^{-1}$ ), possibly due to a higher density of trees (Table 4 and Table 5). In the Sari forest area, the total basal area was higher for Quercus liucotrichophora $\left(4.242 \mathrm{~m}^{2} \mathrm{ha}^{-1}\right)$, possibly due to a higher density of trees (Table 4 and Table 5). In the Gaurikhund forest area, the total basal area was higher for Quercus liucotrichophora $\left(7.319 \mathrm{~m}^{2} \cdot \mathrm{ha}^{-1}\right)$, possibly due to a higher density of trees (Table 4 and Table 5). In the Trijuginarayan forest area, the total basal area was higher for Quercus liucotrichophora $\left(8.89 \mathrm{~m}^{2} \cdot \mathrm{ha}^{-1}\right)$, possibly due to a higher density of trees (Table 4 and Table 5). In the Kedarnath forest area, the total basal area was higher for Taxus wallichiana $\left(4.654 \mathrm{~m}^{2} \cdot \mathrm{ha}^{-1}\right)$, possibly due to a higher density of trees (Table 4 and Table 5). In the Tungnath forest area, the total basal area was higher for Rhododendron barbatum $\left(20.59 \mathrm{~m}^{2} \cdot \mathrm{ha}^{-1}\right)$, possibly due to a higher density of trees (Table 4 and Table 5).

\section{Diversity Index}

The species diversity index (Shannon-Wiener) can be regarded as a measure of environmental quality and points to the well-being of any ecosystem. The plant species diversity indices for site $\mathrm{S} 1$ to $\mathrm{S} 9$ have been presented in Table 5. For site $S_{1}$, it was 3.028 for trees, 2.788 for shrubs, and 3.613 for herbs. However, for site $S_{2}$, it was found to be 3.048 for trees, 2.696 for shrubs, and 3.787 for herbs. For site $S_{3}$, it was 2.901 for trees, 2.629 for shrubs, and 3.531 for herbs. For site $\mathrm{S}_{4}$, it was 3.001 for trees, 2.492 for shrubs, and 3.305 for herbs. For site $S_{5}$, it was 2.918 for trees, 2.594 for shrubs, and 3.1564 for herbs. For site $\mathrm{S}_{6}$, it was 2.753 for trees, 2.404 for shrubs, and 3.367 for herbs. For site $S_{7}$, it was 2.636 for trees, 2.047 for shrubs, and 3.317 for herbs. For site $S_{8}$, it was 1.737 for trees, 0.192 for shrubs, and 3.712 for herbs. For site $\mathrm{S}_{9}$ it was 3.115 for herbs. This pointed out the dominance of herbs and trees at sites $\mathrm{S}_{1}, \mathrm{~S}_{2}$, and $\mathrm{S}_{4}$, and the dominance of herbs at sites $S_{3}, S_{5}, S_{7}, S_{8}$, and $S_{9}$. The dominance of both herbs and shrubs is only at site $S_{6}$. The dominant tree species was Abies spectabilis, whereas, the dominant shrub species was Rhododendron campanulatum.

\section{IMPACTS OF ECODISASTER 2013 ON FOREST AREA OF KEDARNATH VALLEY}

During the study, it was discovered that during the Kedarnath eco-disaster in Kedarnath valley in June 2013, there was a lot of damage to the forest in the riverbank of the Mandakini River due to flash floods and landslides. The flood plain of the Mandakini River was totally destroyed in which several important medicinal plants flowering plants and ornamental plant species were washed. In this disaster, about 500 valuable plant communities were affected. Even in the lower areas of Kedarnath, the nearby forest area of Mandakini River was damaged. Most of the forest was damaged in Jangalchhati, Rambara, Bhimbali, Gaurikund, Sonprayag, and Sitapur, in which medicinal plants, fodder plants, and wild edible plants were completely destroyed. In this disaster, landslides and flash floods that occurred in Kali gad, Madhmaheswar gad, and Kakara gad destroyed forest, in which, many fuel and fodder plants forest area was damaged.

Rawat et al. (2016) studied the biomass estimation during 2012 by sampling at ten random plots laid at open and dense forest sites. The biomass obtained from that study had shown that 242.24 ton.ha ${ }^{-1}$ to 322.97 ton.ha ${ }^{-1}$ for the Mixed Forest. The total washed-out area from the forest was nearly an average of 92.44 (Open and Dense Forest). This showed that nearly 22392.66 to 29855.35 tons of biomass from the total 
area was lost. The disturbance in dense mixed forest (33.16 ha) and open mixed forest ( $59.28 \mathrm{ha}$ ) was recorded by Rawat et al. (2016) (Fig. 2). Over 500 plant species have suffered losses varying from minor to significant. Considering heavy riverbank cutting, multiple landslides event, and deposition of sediments in the Kedarnath pastoral area, the impact on vegetation is comparatively higher in meadows (BSI 2015).

It will take many thousands of years for regeneration in natural conditions for vegetation growth and productivity. To an ecosystem, the biomass and thus carbon sequestration process are directly linked. Loss in biomass from the available species extinction is a greater loss for the ecological cycle from the present area.

\section{Ethnobotanical Plants and Their Use}

Kedarnath valley is very rich in terms of the presence of medicinal plants, Edible plants, Fodder plants, Timber trees and fuelwood, and economically important plants. Local people of the Kedarnath valley use these plants for the cure of several diseases, as fodder, timber, and fuelwood (Table 3). A large number of these species are harvested in the wild, particularly for food, medicinal purposes, and for sale (Prasad \& Sharma 2018).

\section{DISCUSSION}

Species richness in a forest depends on climatic, edaphic, and biotic factors (Ayappan \& Parthasarathy 2001). A total of 221 plant species were recorded in Kedarnath valley. The species diversity of Kedarnath valley was found in the following order Herbs (144)> Trees (49) >Shrubs (28). Semwal et al. (1999) reported a total of 81 plant species including 20 tree species, 24 shrubs species, and 37 herbs species in the forests of Jardhar in Garhwal Himalaya. Kharkwal et al. (2005) carried out a study in the pine forest at different altitudes of Central Himalaya and reported a total of 56 species comprising 51 genera and 28 families, which is lower than the present study. The tree density in the present study was highest in the Kedarnath valley which ranged from 0.3 to 8.5no./ha. Sinha and Maikhuri (1998) also reported almost the same density in core and interactive zones of the Haryali sacred forest of Garhwal Himalaya. Chandrashekara \& Sankar (1998) reported a stem density of the iringole sacred grove in Kerala. These values were within the values reported by Saxena and Singh (1982), Bargali et al. (1988), Pangtey et al. (1989), and Bhandari et al. (1997) for various forests of Garhwal Himalaya. Shrub density in the present study varied from 0.4 to 13.5 no./ha, whereas herb density ranged between 0.2 to 22.4 no./ha. These values are comparable to the reported values of Kumar et al. (2009), Uniyal et al. (2010) for a forest in Garhwal Himalaya. A/F ratio is used to interpret the distribution pattern of species. Odum (1971) stated that clumped (contagious) distribution is the commonest pattern in nature, and random distribution is found only in a uniform environment and the regular distribution occurs where severe competition between the individuals exists (Panchal \& Pandey 2004). Pala et al. (2011) have reported trees, shrubs, and herbs density of 6.88 trees $100 \mathrm{~m}^{-2}, 12.8$ shrubs $25 \mathrm{~m}^{-2}$, and 16.34 herbs $\mathrm{m}^{-2}$ respectively in Chanderbadni sacred forest of Garhwal Himalaya.

Total basal cover (TBC) for trees showed a range of 9.542 to $0.075 \mathrm{~m}^{2}$.ha ${ }^{-1}$ from the Kedarnath valley forest. The variations in the TBC in different study sites may be due to variations in the number and size of tree species in different sites. The present study values are supported by Pande et al. (2001), who observed TBC ranged from between 56.42-126 $\mathrm{m}^{2} \cdot \mathrm{ha}^{-1}$ in a forest in Garhwal Himalaya. Vidyasagaran et al. (2005) reported the average TBC value of $25.79 \mathrm{~m}^{2} \cdot \mathrm{ha}^{-1}$ in sacred groves of the Thrissur district of Kerala. Sinha and Maikhuri (1998) also reported TBC values of 47.59 to 26.87 $\mathrm{m}^{2}$.ha $\mathrm{h}^{-1}$ in the core and interactive zones of the Hariyali sacred forest from Garhwal Himalaya. Sacred forests mostly show reduced forest loss than unprotected areas and higher plant species richness, canopy heights, and stem diameters (Campbell 2004). Rawat (2005) also reported TBC values between $3.74-80.36 \mathrm{~m}^{2} /$ ha for temperate forests in Garhwal Himalaya. Tripathi and Singh (2009) reported that basal area is an important indicator of tree stocking, which reflects stand volume or biomass and recorded $24.84 \mathrm{~m}^{2} \cdot \mathrm{ha}^{-1}$ basal areas of trees in a riverine forest of Katernia ghat Wildlife Sanctuary.

Shannon diversity index $(\bar{H})$ for tree species was recorded from a minimum of 0.976 to a maximum of 3.048 in $\mathrm{Ke}$ darnath valley. The values of the present study were higher than the values (1.44-2.27) calculated by Looy et al. (2003) on the effect of river embankment and forest fragmentation on plant species and composition of flood plain forests in the Meuse valley, Belgium. The values of the present study were higher than the values $(0.8-1.4)$ reported by Pala et al. (2011) in the forests along the river Ganga in the Himalayas. Shannon Wiener diversity index $(\bar{H})$ for shrub species was recorded from lowest (0.192) to highest (2.788) in the Kedarnath valley. Ram et al. (2004) reported shrub diversity from 2.6 to 3.8 for different forest types in Kumaun Himalaya. Shannon Wiener's diversity index $(\bar{H})$ for herb species was recorded from minimum (3.115) to maximum (3.787) in Kedarnath valley. The values of the present study were within the values reported for different forests by many workers (Singh \& Singh, 1986, Pande et al. 2002). The values of the present study are also within the reported values (3.24 to 4.03) given by Kharkwal et al. (2005).

Several workers (Greig-Smith 1957, Singh \& Yadav 1974) have reported contagious distribution in natural veg- 


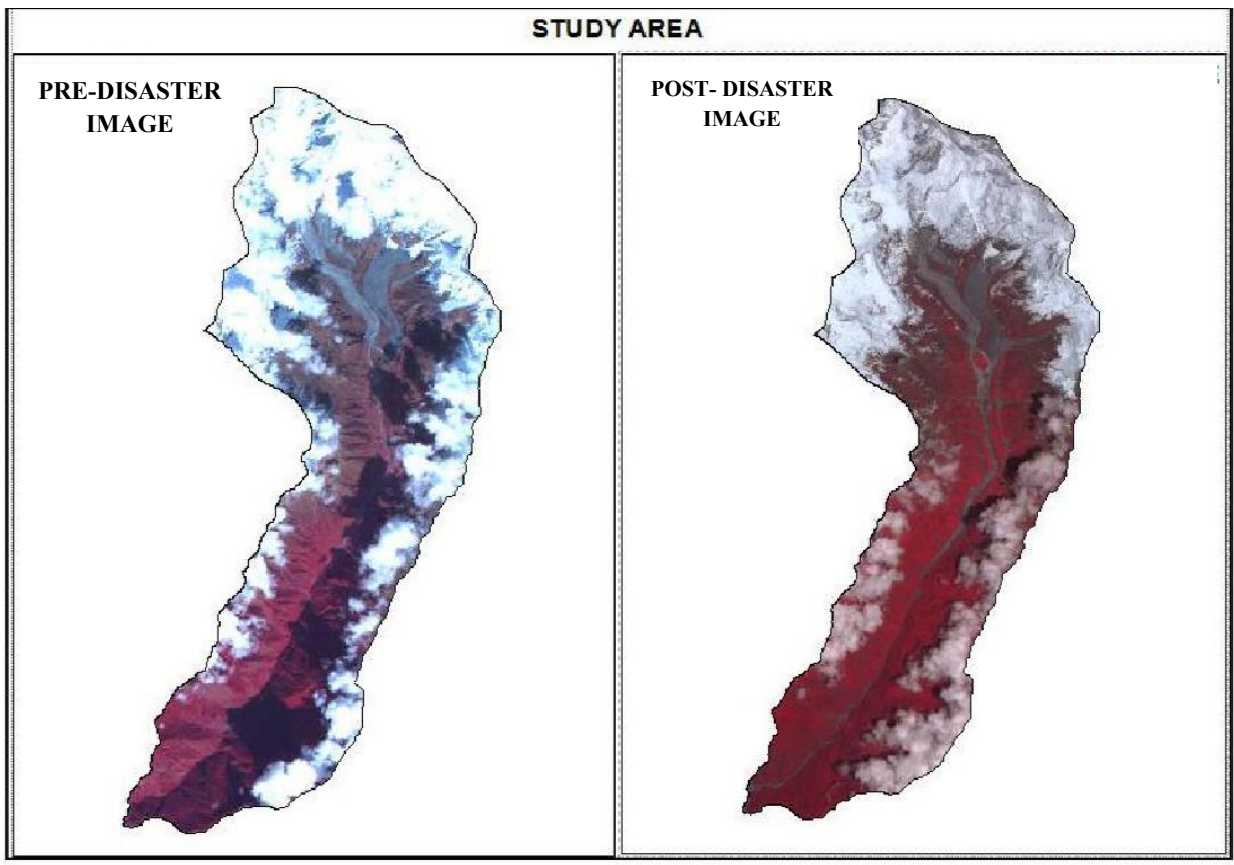

Pre Disaster

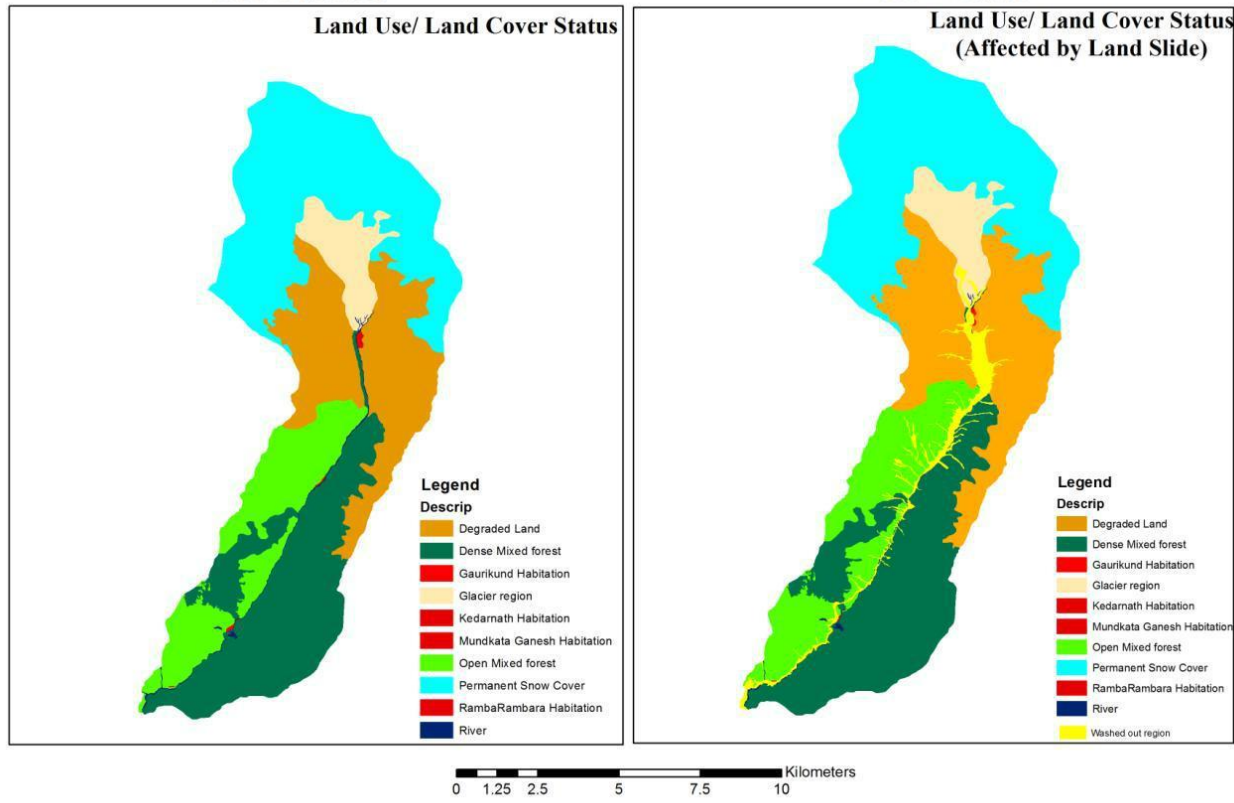

Fig. 2: Satellite imageries: Landuse landcover status of the study area pre and post-disaster of Kedarnath valley.

etation. However, shrubs and herbs were found distributed contagiously in all study sites. The regular distribution pattern was entirely absent. Mishra and Laloo (2005) and Upadhaya et al. (2004) also reported a contagious pattern of distribution for subtropical forests of North-east India. Other studies conducted within Garhwal Himalaya (Bhandari et al. 1998, Pande et al. 2002) have also shown a contagious pattern of
Vegetational distribution in different forest types. Rawat et al. (2018) studied tree species richness, dominance, and regeneration status in western Ramganga valley. Bhatt et al. (2020) worked on God's tree: A culturally coded strategy for conservation in Chamoli District. Tiwari et al. (2020) also worked on weed floristic composition and diversity in paddy fields of Mandakini valley. 


\section{CONCLUSION}

The current study documented that the Kedarnath valley is blessed with mainly eight types of forests that include the Himalayan Dry Temperate Forests, Dry Temperate Coniferous Forest, West Himalayan Birch/Fir Forests, Sub-alpine Pasture, Himalayan Chir-Pine Forest, Himalayan Moist Temperate Forest, West Himalayan Sub-Alpine Birch/Fire Forest, and Alpine Forest. The largest forest cover was found in Karokhi followed by Sari, Ransi, Ukhimath, Kabiltha, whereas, the lowest forest cover was recorded in Tungnath and Barasu. A total number of 221 plant species were collected and documented from the Kedarnath valley. Plant diversity in the valley encompasses 49 species of trees, 28 species of shrubs, and 144 species of herbs. The tree density in the current study was recorded highest in the Kedarnath valley which ranged from 0.3 to 8.5 no.ha ${ }^{-1}$ Shrub density in the present study varied from 0.4 to 13.5 no.ha $^{-1}$, whereas herb density ranged between 0.2 to 22.4 no.ha ${ }^{-1}$. Total basal cover (TBC) for trees showed a range from 9.542 to 0.075 $\mathrm{m}^{2} /$ ha, Total basal cover (TBC) for trees showed a range of 9.542 to $0.075 \mathrm{~m}^{2} \cdot \mathrm{ha}^{-1}$, and the Shannon diversity index $(\bar{H})$ for tree species was recorded from a minimum of 0.976 to a maximum of 3.048 .

The Kedarnath valley consisted of patchy vegetation including many economically important plants such as medicinal herbs, timber trees wild edible plants, fodder, and fuelwood. During the Kedarnath eco-disaster that occurred in June 2013, huge damage to the forest in the riverbank of the Mandakini River was recorded due to flash floods and landslides. It was estimated that nearly 500 valuable plant species were affected by this eco-disaster.

\section{ACKNOWLEDGEMENTS}

The authors are thankful to the local people of the Kedarnath valley and VDO of tehsil Ukhimath to share their valuable knowledge, data and help in data collection. One of the authors (Chandi Prasad) thankfully acknowledges the H.N.B. Garhwal University (A Central University) for providing support during the research.

\section{REFERENCES}

Ayyappan, N. and Parthasarathy, N. 2001. Patterns of tree diversity within a large-scale permanent plot of tropical evergreen forest Western Ghats, India. Ecotropica, 5: 197-211.

Ballabha, R., Rawat, D.S., Tiwari, J.K., Tiwari, P. and Gairola, A. 2013. Wild edible plant resources of the Lohba Range of Kedarnath Forest Division (KFD), Garhwal Himalaya, India. Int. Res. J. Biol. Sci., 2(11): 65-73.

Bargali, K., Usman and Joshi, M. 1998. Effect of forest covers on certain site and soil characteristics in Kumaun Himalayas. Indian J. For., 21(3): 224-227.
Bhandari, B.S., Mehta, J.P., Nautiyal, B.P. and Tiwari, S.C. 1997. Structure of a chir pine (Pinus roxburghii Sarg.) community along an altitudinal gradient in Garhwal Himalaya. Int. J. Ecol. Enviro. Sci., 23(1): 67-74.

Bhandari, B.S., Mehta, J.P. and Tiwari, S.C. 1998. Woody vegetation structure along an altitudinal gradient in a part of Garhwal Himalaya. J. Hill Res., 11: 26-31.

Botanical Survey of India. 2015. Kedarnath natural disaster impact on flora. MoEFCC, pp. 1-40.

Bhatt, V.P. and Rawat, D.S. 2020. God's Tree: A Culturally Coded Strategy for Conservation (A Case Study of Gairsain Ecoregion of District Chamoli. Uttarakhand). In: Khasim, S.M., Long, C., Thammasiri, K. and Lutken, H. (eds.) Medicinal Plants: Biodiversity, Sustainable Utilization, and Conservation, Springer, New York, pp. 237-247.

Campbell, M. O. 2004. Traditional forest protection and woodlots in the coastal savannah of Ghana. Environ. Conserv., 31: 225-232.

Census of India. 2011. District Census Handbook Rudraprayag. Directorate of Census Operation, Uttarakhand, pp. 1-156.

Chandrashekara, U. M. and Sankar, S. 1998. Ecology and management of sacred groves in Kerala. For. Ecol. Manag., 112: 165-177.

Curtis, J.T. 1951. An upland forest continuum in the prairie forest border region of Wisconsin. Ecology, 32: 476-496.

Curtis, J.T. 1959. The Vegetation of Wisconsin. An Ordination of Plant Communities. University Wisconsin Press, Wisconsin.

Curtis, J.T. and Mc-Intosh, R.P. 1950. The interrelations of certain analytic and synthetic phtyosociological characters. Ecology, 32: 434-455.

FSI, 2017. Forest Survey of India. India State of Forest Report. Ministry of Environment and Forests, Dehradun, pp. 308-313.

Gates, F. C. 1949. Field Manual of Plant Ecology. Mc Graw Hill, New York. Greig-Smith, P. 1957. Quantitative Plant Ecology. Butterworths, London.

Kharkwal, G., Mehrotra, P. and Pangtey, Y.P.S. 2005. Comparative studies on species richness, diversity, and composition of oak forests in Nainital district, Uttaranchal. Curr. Sci., 89(4): 668-676.

Kimothi, G.P. and Shah, B.C.L. 1989. Some medicinal plants of Gopeshwar-Tungnath region of Uttar Pradesh. Anc. Sci. of Life, 8(3,4): 283-292.

Kumar, B. 2009. Major religious plants of Rudraprayag District (Garhwal), Uttarakhand (India), Ethnobot. Leaflets, 13:1476-1484.

Looy, K.V., Honnay, O., Bossuyt, B. and Hermy, M. 2003. The effects of river embankment and forest fragmentation on the plant species richness and composition of floodplain forests in the Meuse Valley. Belgium. Belg. J. Bot., 136 (2): 97-108.

$19^{\text {th }}$ Livestock Census. 2012. All India report. Ministry of Agriculture Department of Animal Husbandry, Dairying \& Fisheries Krishi Bhawan, New Delhi, pp. 1-120.

Mishra, B.P. and Laloo, R.C. 2005. Effect of fragmentation on plant diversity and community characters of the sacred grove of Meghalaya. In: National Conference on Current Trends of Research in Science and Technology 50th Annual Technical Session of Assam. Science Society Deka PC, Jha DK Assam Science Society Guwahati.

Misra, R. 1969. Ecology Workbook. Oxford and IBH, Calcutta, p. 244.

Misra, R. and Puri, G.S. 1954. Indian Manual of Plant Ecology. English Book Depot, Dehradun.

Mullar-Dombois, D. and Ellenberg, H. 1974. Aims and Methods of Plant Ecology. John Wiley and Sons, New York.

Nautiyal, M. and Tiwati, J.K., Rawat, D.S. 2017. Exploration of some important fodder plants of Joshimath area of Chamoli district of Garhwal, Uttarakhand. Curr Bot., 8: 144-149.

Negi, B.S., Chauhan, D.S. and Todariya, N.P. 2008. Inventory of species richness of panchayat forests and adjoining reserve forests in three districts of Garhwal Himalaya, India. Int. Soc. Trop. Ecol., 49(2): 121-129.

Odum, E.P. 1971. Analysis of vegetation of Rampara forest in Saurashtra region of Gujarat state of India. J. Trop. Ecol., 45(2), 223-231.

Pala, N.A., Negi, A.K., Gokhale, Y. and Todaria, N.P. 2011. Species composition and phytosociological status of Chanderbadni sacred forest in Garhwal Himalaya Uttarakhand India. NeBIO, 2: 52-59. 
Pala, N.A., Negi, A.K., Gokhale, Y.S. and Kumar, M. 2016. Community structure and plant diversity of community-based religious conserved forest of Garhwal Himalaya, India. J. Earth. Sci. Clim Change, 7(2): 334.

Panchal, N. and Pandey, A. N. 2004. Analysis of vegetation of Rampara forest in Saurashtra region of Gujarat state of India. Trop. Ecol., 2)45): 223-231.

Pande, P. K., Negi, J. D. S. and Sharma, S. C. 2001. Plant species diversity and vegetation analysis in moist temperate Himalayan forest. Indian Journal of Forestry, 24: 456-470.

Pande, P. K., Negi, J. D. S. and Sharma, S. C. 2002. Plant species diversity, composition, gradient analysis, and regeneration behavior of some tree species in a moist temperate western Himalayan forest ecosystem. Indian Forester, 128: 869-889.

Pangtey, Y. P. S., Samant, S. S., Bankoti, N. S. and Rawal, R. S. 1989. Soil and vegetation analysis of Pindari area. Second Annual report submitted to Department of Environment, New Delhi, pp. 167.

Kumar, M., Singh, B., Joshi, M. 2009. Effect of aspect on distribution pattern of Anogeissus latifolia Wall ex Bedd. in subtropical belt of Garhwal Himalaya, India. Tanzania J. For. Nat. Conserv., 78(1): 21-27.

Phillips, E. A. 1959. Methods of Vegetation Study. Henry Holt. \& Co., Inc., New York.

Prasad, C. and Sharma, R.C. 2018. Wild edible resources of Kedarnath valley, Garhwal Himalaya, India. Indian J. Ecol., 45(3): 433-444.

Ram, J., Kumar, A. and Bhatt, J. 2004. Plant diversity in six forest types of Uttaranchal, Central Himalaya, India. Curr. Sci., 86(7): 975-978.

Rawat, N., Thapliyal, A., Purohit, S., Negi, G.S., Dangwal, S., Rawat, S., Aswal, A. and Kimothi, M.M. 2016. Vegetation loss and ecosystem disturbances on Kedargad Mandakini subwatershed in Rudraprayag district of Uttarakhand due to torrential rainfall during June 2013. Int. J. Adv. Remote Sens. GIS, 5(4): 1662-1669.

Rawat, R.S. 2005. Studies on the interrelationship of woody vegetation density and soil characteristics along an altitudinal gradient in a montane forest of Garhwal Himalayas. Indian For., 131: 990-994.

Rawat, D.S., Tiwari, J.K., Tiwari, P., Nautiyal, M., Praveen, N. and Singh, N. 2018. Tree species richness, dominance, and regeneration status in western Ramganga Valley, Uttarakhand Himalaya, India. FRI, Dehradun, 144(7): 595-603.

Revenue Report of Villages. 2017. Revenue Report of Villages Tehsil Ukhimath: 2016-17 R-57. Ukhimath Tehsil, Rudraprayag district, Uttarakhand, pp. 1-12.

Saxena, A.K. and Singh, J.S. 1982. A phytosociological analysis of woody species in forest communities of a part of Kumaun Himalaya. Vegetatio, 50: 3-22.
Semwal, D.P., Saradhi, P.P., Kala, C.P. and Sajwan, B.S. 2010. Medicinal plants used by local Vaidyas in Ukhimath block, Uttarakhand. Indian J. Tradit. Knowl., 9(3): 480-485.

Semwal, R.L., Nautiyal, S., Rao, K.S., Maikhuri, R.K. and Bhandari, B.S. 1999. Structure of Forests under community conservation: A preliminary study of Jardhar village initiative in Garhwal Himalaya. Envis, 7(2): 20-31.

Singh, A., Nautiyal, M.C., Kunwar, R.M. and Bussmann, R.W. 2017. Ethnomedicinal plants used by local inhabitants of Jakholi block, Rudraprayag district, western Himalaya, Indian J. of Ethnobiol. Ethnomed., 13: 1-29.

Singh, J.S. and Yadav, P.S. 1974. Seasonal variation in composition plant biomass and net primary productivity of tropical grassland at Kurukshetra, India. Ecol. Monogr., 44: 351-376.

Singh, S.P. and Singh, J.S. 1986. Structure and function of Central Himalayan oak forest. Proc. Indian Acad. Sci.: Plant Sci., 96: 159-189.

Sinha, B. and Maikhuri, R.K. 1998. Conservation through socio-cultural religious practice in Garhwal Himalaya: A case study of Hariyali sacred site. In:Ramakrishnan, P.S., Saxena, K.G. and Chandrashekara U.M. (eds.). Conserving the Sacred for Biodiversity Management. UNESCO and Oxford-IBH Publishing, New Delhi, pp. 289-299.

Srivastava, S. K. and Singh, D. K. 2005. Glimpses of the Plant Wealth of Uttaranchal. Bishen Singh Mahendra Pal Singh, Dehradun, pp. 158

Tiwari, P., Bharti, Rawat, D. S. and Singh, N. 2020. Weed floristic composition and diversity in paddy fields of Mandakini Valley, Uttarakhand, India. Int. J.Bot. Stud., 5(3): 334-341.

Tripathi, K.P. and Singh, B. 2009. Species diversity and vegetation structure across various strata in natural and plantation forests in Katerniaghat wildlife sanctuary, North India. Trop. Ecol., 50(1):191-200.

Uniyal, B.P., Sharma, J.R., Chaudhari, U. and Singh, D.K. 2007. Flowern ing Plants of Uttarakhand: A Checklist. Bishan Singh Mahendra Pal. Singh, Dehradun, pp. 404-1.

Uniyal, P., Pokhriyal, P., Dasgupta, S., Bhatt, D. and Todaria, N.P. 2010. Plant diversity in two forest types along the disturbance gradient in Dewalgarh watershed, Garhwal Himalaya. Curr. Sci., 98 (7): 938-943.

Upadhaya, K., Pandey, H.N., Law, P.S. and Tripathi, R.S. 2004. Diversity and population characteristics of woody species in sub-tropical humid forests exposed to culture disturbances in Meghalaya NE India. Trop. Ecol., 45: 303-314.

Vidyasagaran, K., Abhilash, D. and Babu, L.C. 2005. Plant diversity and conservation of Kalasamala sacred grove of Thrissur District Kerala. In: Kunihikannan, C. and Singh, B.G. (eds.). Strategy for conservation of sacred groves. Institute of Forest Genetics and Tree Breeding, Coimbatore, pp. 77-81. 